\title{
LATTICE BOLTZMANN SIMULATION OF DROPLET BEHAVIOUR IN MICROFLUIDIC DEVICES ${ }^{1}$
}

\author{
Haihu Liu and Yonghao Zhang ${ }^{2}$ \\ Department of Mechanical Engineering, University of Strathclyde, Glasgow G1 1XJ, UK
}

KEY WORDS: droplet dynamics, lattice Boltzmann method, phase field, boundary condition.

\begin{abstract}
We developed a lattice Boltzmann model to investigate the droplet dynamics in microfluidic devices. In our model, a stress-free boundary condition was proposed to conserve the total mass of flow system and improve the numerical stability for flows with low Reynolds number. The model was extensively validated by the benchmark cases including the Laplace's law, the static contact angles at solid surface, and the droplet deformation and breakup under simple shear flow. We applied out model to study the effects of the Pelcect number, the Capillary number and wettability on droplet formation. The results showed that the Peclet number has little effect on droplet size though it slightly affects the time of droplet formation. In the screeping flow regime, the Capillary number plays a dominating role in thedroplet generation process. Wettability of fluids affects the position of droplet detachment, the droplet shape and size, and its impact becomes more significant when the Capillary number decreases. We also found that the hydrophobic surface generally can produce smaller droplet.
\end{abstract}

\section{INTRODUCTION}

Microdroplet technology is emerging to transform current biological and chemical processing in laboratories which is critical to the healthcare, food, chemical and pharmaceutical industries [1,2]. T-junction is often used to produce immiscible microdroplets [3,4], where many factors, including the interfacial tension, viscosity, the inlet velocities of the two fluids and the wetting properties of solid walls, will affect droplet generation, transportation and interaction. Therefore, understanding droplet behaviour in microchannel becomes esstential in device optimal design and operation. However, it is challenging to numerically simulate microdroplet generation, transportation, coalescence and interaction with solid surface. Front tracking methods are not suitable for simulating droplet breakup and coalescence, while interface capture methods such as Volume-of-Fluid (VOF) and level set models will experience numerical instability at the interface region when the surfacial tension is dominant in interfacial dynamics for small droplet [5]. Recently developed lattice Boltzmann (LB) method is mesoscopic method that can provide many advantages of molecular dynamics, making the LB method especially useful for simulation of droplet dynamics [6]. It is a pseudo-molecular method tracking evolutions of the distribution function of assembly of molecules and built upon microscopic models and mesoscopic kinetic equations [7].

Currently, the most applied LB multiphase models are the colour-function model [8], the pseudo-potential model [9] and the free energy model [10,11]. The colour function model is similar to the VOF method in terms of modeling interface. The pseudo-potential model only considers the nearest neighbour interactions so that the only thermodynamically consistent choice of the potential will lead to "mass collapse" while the other choice of the potential will cause numerical instability [12]. In the free energy model, the phase field description of interface has been adopted where a convection and diffusion equation for an order parameter is solved to describe the interface evolution. This order parameter is not merely a convenient parameter for computation purpose as the colour function, it depends on thermodynamic properties and is controlled by the local free energy. However, the free energy model violates the Galilean invariance and is not thermodynamically consistent for non-ideal gases [6]. In addition, minimising unphysical spurious velocities at interface remains a challenge for free energy LB models. Because the pressure gradients in the interfacial regions are usually small, numerical errors due to violation of the Galilean invariance in the free energy model are insignificant [11]. In addition, one advantage of the free energy model is its implementation of

\footnotetext{
${ }^{1}$ This work was presented in the $1^{\text {st }}$ European Conference on Microfluidics, Bologna, Italy, December 10-12, 2008

2 Email: yonghao.zhang@strath.ac.uk
} 
microscopic physics so that the interface structure is resolved, whereas both the colour-function model and the pseudo-potential model lack a clear relation to classical thermodynamics. Finally, microscopic interactions can be easily incorporated in the model so that it is very convenient to study the effect of the molecular level interaction in interfaces on macroscopic flow. Consequently, we use the free energy LB model in this paper.

Boundary treatment is one of the key issues in the LB modelling interfacial flows. In the commonly-used boundary treatments [6], the unknown distributions at the boundaries are determined by the local properties, such as density and velocity. Therefore, we need accurate solutions of the local properties. In general, the simplest and most convenient way to impose the outflow boundary is to extrapolate the distribution functions at the boundaries. However, Chikatamarla et al. [13] pointed out that this boundary treatment usually causes numerical instability. The unreasonable errors start at the outlet and propogate upstream, especially for the flow with small Reynolds number. Tong et al. [14] argued that the main reason of instability lies in which the whole mass conservation cannot be satisfied in the computational domain. Aiduny et al. [15] developed a stress-free outflow boundary treatment, which can conserve the total mass of system. However, their method applies uniform modification to the particle distribution functions for the rest particles which neglects the effect of the velocity difference at the outlet and thus leads to the numerical instability especially for creeping flows. Here, we propose an improved mass modification to take account of the effect of fully developed velocity profile, which can ensure the numerical stability especially for creeping flows. With an improved stress-free outflow boundary treatment, we will study the influences of the Peclet number, Capillary number and wettability of fluids on droplet formation to achieve a better understanding of droplet behaviour in a T-junction microfluidic device.

\section{NUMERICAL METHOD}

\subsection{Lattice Boltzmann Scheme}

In the free energy model $[10,11]$, two sets of distribution functions $f_{i}(\mathbf{x}, t)$ and $g_{i}(\mathbf{x}, t)$ are used to the distribution functions for the velocity field $\mathbf{u}(\mathbf{x})$ and the order parameter field $\phi(\mathbf{x})$ respectively, and their evolution are governed by the lattice Bhatnagar-Gross-Krook (BGK) equations:

$$
\begin{aligned}
& f_{i}\left(\mathbf{x}+\mathbf{e}_{i} \Delta t, t+\Delta t\right)-f_{i}(\mathbf{x}, t)=-\frac{1}{\tau_{f}}\left[f_{i}(\mathbf{x}, t)-f_{i}^{e q}(\mathbf{x}, t)\right], \\
& g_{i}\left(\mathbf{x}+\mathbf{e}_{i} \Delta t, t+\Delta t\right)-g_{i}(\mathbf{x}, t)=-\frac{1}{\tau_{g}}\left[g_{i}(\mathbf{x}, t)-g_{i}^{e q}(\mathbf{x}, t)\right],
\end{aligned}
$$

where $\tau_{f}$ and $\tau_{g}$ are the independent relaxation parameters, and $f_{i}^{e q}(\mathbf{x}, t)$ and $g_{i}^{e q}(\mathbf{x}, t)$ are local equilibrium distribution functions at position $\mathbf{x}$ and time $t$. The label $i$ denotes a particular lattice vector $\mathbf{e}_{i}$, defined by $\mathbf{e}_{0}=(0,0), \mathbf{e}_{1,3}=( \pm c, 0), \mathbf{e}_{2,4}=(0, \pm c), \mathbf{e}_{5,7}=( \pm c, \pm c)$ and $\mathbf{e}_{6,8}=(\mp c, \pm c)$ in the D2Q9 model [16]. The lattice velocity parameter $c$ is defined by the lattice size $\Delta x$ and the time step $\Delta t$, i.e. $c=\Delta x / \Delta t$.

The underlying physical properties of LB schemes are determined via the hydrodynamic moments of the equilibrium distribution functions. The moments of the distribution functions should satisfy

$$
\begin{gathered}
\sum_{i} f_{i}=\sum_{i} f_{i}^{e q}=\rho, \quad \sum_{i} g_{i}=\sum_{i} g_{i}^{e q}=\phi, \\
\sum_{i} f_{i} e_{i \alpha}=\sum_{i} f_{i}^{e q} e_{i \alpha}=\rho u_{\alpha}, \quad \sum_{i} g_{i} e_{i \alpha}=\sum_{i} g_{i}^{e q} e_{i \alpha}=\phi u_{\alpha}, \\
\sum_{i} f_{i}^{e q} e_{i \alpha} e_{i \beta}=P_{\alpha \beta}+\rho u_{\alpha} u_{\beta}, \quad \sum_{i} g_{i}^{e q} e_{i \alpha} e_{i \beta}=\Gamma \mu \delta_{\alpha \beta}+\phi u_{\alpha} u_{\beta}, \\
\sum_{i} f_{i}^{e q} e_{i \alpha} e_{i \beta} e_{i \gamma}=\rho c_{s}^{2}\left(u_{\alpha} \delta_{\beta \gamma}+u_{\beta} \delta_{\alpha \gamma}+u_{\gamma} \delta_{\alpha \beta}\right),
\end{gathered}
$$


where $\rho$ is the total density, $P_{\alpha \beta}$ is the pressure tensor, $c_{s}$ is the speed of sound and isotropy of viscosity requires that $c_{s}=c / \sqrt{3}$ [6]. The order parameter $\phi$ is chosen to be the normalized density difference of the two fluids, and $\Gamma$ is a coefficient related to the mobility of the fluid, $M$, which is

$$
M=\Gamma\left(\tau_{g}-1 / 2\right) \Delta t .
$$

Based on the above constraints of Eqs. (3)-(6) and to reduce the magnitude of spurious velocities near the interface, the equilibrium distribution functions can be written as [17]

$$
\begin{aligned}
f_{i}^{e q}= & \frac{w_{i}}{c^{2}}\left[p-\frac{\kappa}{2} \nabla^{2} \phi+e_{i \alpha} \rho u_{\alpha}+\frac{3}{2 c^{2}}\left(e_{i \alpha} e_{i \beta}-c_{s}^{2} \delta_{\alpha \beta}\right) \rho u_{\alpha} u_{\beta}\right] \\
& +\frac{\kappa}{c^{2}}\left(w_{i}^{x x} \partial_{x} \phi \partial_{x} \phi+w_{i}^{x y} \partial_{x} \phi \partial_{y} \phi+w_{i}^{y y} \partial_{y} \phi \partial_{y} \phi\right), \\
g_{i}^{e q} & =\frac{w_{i}}{c^{2}}\left[\Gamma \mu+e_{i \alpha} \phi u_{\alpha}+\frac{3}{2 c^{2}}\left(e_{i \alpha} e_{i \beta}-c_{s}^{2} \delta_{\alpha \beta}\right) \phi u_{\alpha} u_{\beta}\right],
\end{aligned}
$$

for $i=1, \ldots, 8$, where $w_{1-4}=\frac{1}{3}, w_{5-8}=\frac{1}{12}, w_{1,3}^{x x}=w_{2,4}^{y y}=\frac{1}{3}, \quad w_{2,4}^{x x}=w_{1,3}^{y y}=-\frac{1}{6}, \quad w_{5-8}^{x x}=w_{5-8}^{y y}=-\frac{1}{24}$, $w_{1-4}^{x y}=0, w_{5,7}^{x y}=\frac{1}{4}$, and $w_{6,8}^{x y}=-\frac{1}{4}$. The other unknown parameters will be discussed in the following section. The equilibrium distributions for the rest particle can be obtained due to the mass conservation as: $f_{0}^{e q}=\rho-\sum_{i \neq 0} f_{i}^{e q}$, and $g_{0}^{e q}=\phi-\sum_{i \neq 0} g_{i}^{e q}$.

\subsection{Free energy model}

The free energy we choose to describe a binary fluid is [18]

$$
F=\int f d \mathbf{x}=\int d \mathbf{x}\left[\rho c_{s}^{2} \ln \rho-\frac{A}{2} \phi^{2}+\frac{B}{4} \phi^{4}+\frac{\kappa}{2}(\nabla \phi)^{2}\right],
$$

where $\kappa$ is a coefficient relating to surface tension and interface thickness. The coefficients $\mathrm{A}$ and $\mathrm{B}$ determine the properties of the bulk phases, and the gradient term is related to the surface tension. The chemical potential difference between the two fluids is given by

$$
\mu=\frac{\partial f}{\partial \phi}-\nabla \cdot \frac{\partial f}{\partial \nabla \phi}=-A \phi+B \phi^{3}-\kappa(\nabla \phi)^{2}
$$

and the corresponding pressure tensor $P_{\alpha \beta}$ can be derived via the Gibbs-Duhem equality [18]:

$$
P_{\alpha \beta}=\phi \mu-f=p \delta_{\alpha \beta}-\kappa\left[(\nabla \phi)^{2} \delta_{\alpha \beta}-\partial_{\alpha} \phi \partial_{\beta} \phi\right],
$$

where the pressure is calculated by

$$
p=\rho c_{s}^{2}-\frac{1}{2} A \phi^{2}+\frac{3}{4} B \phi^{4}-\kappa \phi \nabla^{2} \phi+\frac{\kappa}{2}(\nabla \phi)^{2} .
$$

Following the same procedure described in [19], we can obtain the order parameter profile along the normal direction of the interface

$$
\phi=\phi_{0} \tanh (x / \xi) \text {. }
$$

Here, $\phi= \pm \phi_{0}$ is the value of the order parameter in the bulk phase at either side of the interface. Assuming $\nabla \phi=0$ in Eq. (11), we can obtain:

$$
\phi_{0}=\left(\frac{A}{B}\right)^{1 / 2}
$$

The thickness of interface layer $\xi$ is:

$$
\xi=\left(\frac{2 \kappa}{A}\right)^{1 / 2}
$$

The interfacial tension becomes: 


$$
\sigma=\frac{4 \kappa}{3 \xi} \phi_{0}^{2}
$$

The local relaxation time can be calculated by the kinmatic viscosity of fluids $v$ :

$$
v=\left(\tau_{f}-1 / 2\right) c_{s}^{2} \Delta t
$$

To solve the pressure tensor and chemical potential, we need to calculate the Laplacian and gradients of the order parameter, which cannot be directly computed from the particle distribution function. Therefore, we use ( 9 points) regular finite difference stencils to compute these derivatives [17]

$$
\partial_{\alpha} \phi=\frac{1}{\Delta t} \sum_{i} w_{i} \phi\left(\mathbf{x}+\mathbf{e}_{i} \Delta t\right) e_{i \alpha}, \quad \nabla^{2} \phi=\frac{2}{\Delta t^{2}} \sum_{i} w_{i}\left[\phi\left(\mathbf{x}+\mathbf{e}_{i} \Delta t\right)-\phi(\mathbf{x})\right] .
$$

\subsection{Boundary conditions}

No-slip boundary condition is applied for all the solid walls using mid-link bounceback [6], which conveniently resolves the complex wall shapes and ensures no "mass leakage". To conserve the total mass of system, a stress-free boundary condition is proposed to treat outflow boundary [15]. The mass conservation of system is satisfied by uniformly modifying the particle distribution functions of the rest particles at the outlet, which can incur numerical instability for the flows with small Reynolds number due to the effect of velocity distribution. In this paper, we introduce the mass modification by considering the fully developed velocity profile to ensure the numerical stability.

Assuming that fluid node $\mathbf{x}$ is a boundary node, whose links are divided into two groups: boundary links (BL) and fluid links (FL) [20]. Without losing generality, the outlet boundary is set at the right-hand end of the system, $x=x_{\text {max }}$; the inlet boundary is set at the left-hand end, $x=x_{\min }$; and the inlet velocity of fluid is directed to the right. After the collision step, the distribution function at the inlet and outlet boundaries is first modified as

$$
\tilde{f}_{i}(\mathbf{x}, t+\Delta t)= \begin{cases}f_{i^{*}}\left(\mathbf{x}, t^{+}\right), & \text {if }\left(i^{*}\right) \text { is } B L, \\ f_{i}\left(\mathbf{x}+\mathbf{e}_{i^{*}}, t^{+}\right), & \text {otherwise, }\end{cases}
$$

where $\mathbf{e}_{i^{*}}=-\mathbf{e}_{i}, t^{+}$is the time immediately after the collision, that is $t^{+}-t<<\Delta t$.

The stress-free outlet boundary can be implemented by

$$
f_{i}(\mathbf{x}, t+\Delta t)= \begin{cases}\tilde{f}_{i}\left(\mathbf{x}^{\prime}, t+\Delta t\right), & \text { if }\left(i^{*}\right) \text { is } B L, \\ \tilde{f}_{i}(\mathbf{x}, t+\Delta t)+\delta f(\mathbf{x}, t+\Delta t), & \text { if } i=0, \\ \tilde{f}_{i}(\mathbf{x}, t+\Delta t), & \text { otherwise }\end{cases}
$$

where $\mathbf{x}^{\prime}=\left(x_{\max }-\Delta x, y\right), \delta f(\mathbf{x}, t+\Delta t)$ will be defined below.

At the inlet boundary, the prescribed constant velocity, $\mathbf{u}_{i n}$, normal to the boundary is imposed by

$$
f_{i}(\mathbf{x}, t+\Delta t)= \begin{cases}\tilde{f}_{i}(\mathbf{x}, t+\Delta t)+2 \rho w_{i} u_{i n}, & \text { if }\left(i^{*}\right) \text { is } B L, \\ \tilde{f}_{i}(\mathbf{x}, t+\Delta t), & \text { otherwise. }\end{cases}
$$

Hence, the total net flux at all lattice sites at the inlet boundary follows

$$
m_{t o t}=\sum_{x=x_{\min }} \sum_{(i *) \in B L} 2 \rho w_{i} u_{i n}=\sum_{x=x_{\min }} \rho u_{i n} .
$$

Assuming that the fully developed velocity profile at the outlet is $\mathbf{u}(\mathbf{x})=(u(y), 0)$, the outgoing mass at site $\left(x_{\max }, y\right)$ can be calculated by

$$
m(\mathbf{x})=m_{t o t} \frac{u(y)}{\sum u(y)} .
$$

In order to conserve the total mass in the system, $\delta f$ in Eq. (21) should be

$$
\delta f(\mathbf{x}, t+\Delta t)=\sum_{\left(i^{*}\right) \in B L}\left[\tilde{f}_{i}(\mathbf{x}, t+\Delta t)-f_{i}\left(\mathbf{x}^{\prime}, t+\Delta t\right)\right]-m(\mathbf{x}) .
$$


For $g_{i}$, a similar bounce-back rule is used at inlet and wall, but a second-order extrapolation scheme is applied at the outlet, namely

$$
g_{i}(\mathbf{x}, t+\Delta t)=2 g_{i}\left(\mathbf{x}^{\prime}, t+\Delta t\right)-g_{i}\left(\mathbf{x}^{\prime \prime}, t+\Delta t\right),
$$

where $\mathbf{x}^{\prime \prime}=\left(x_{\max }-2 \Delta x, y\right)$.

For the interaction of immiscible fluids with solid walls, one has to account for the wetting properties. Iwahara et al. [21] proposed a very elegant way of implementing the wetting boundary condition in a vapor/liquid system. Recently, van der Graaf et al. [22] applied the same wetting boundary treatment in a liquid/liquid system. They both assume that the wall is a mixture of two fluids, thus having a certain value of the order parameter $\phi_{w}$. This value is used to compute the derivatives of the order parameter using the finite difference stencils as described above. Similarly, we use the following formula to assign order parameter $\phi_{w}$ to the solid lattice site next to the wall.

$$
\cos (\theta)=\frac{1}{2} \tilde{\phi}\left(3-\tilde{\phi}^{2}\right)
$$

where $\tilde{\phi}=\phi_{w} / \phi_{0}, \theta$ is the desired equilibrium contact angle, which is related to interfacial tensions by the Young's equation:

$$
\cos (\theta)=\frac{\sigma_{o s}-\sigma_{w s}}{\sigma}
$$

where $\sigma_{o s}$ and $\sigma_{w s}$ are the interfacial tensions between the fluid phase (oil and water) and the solid wall.

\section{RESULTS AND DISCUSSIONS}

\subsection{Validation of the model}

We first test the capability of the model to predict the Laplace's law for surface tension. We construct a $256 \times 256$ domain with a droplet centred in the middle of the geometry and measure the pressure difference across the droplet interface. The Laplace's law is given as

$$
p_{i}-p_{o}=\sigma / R
$$

where $p_{i}$ and $p_{o}$ are the pressure inside and outside the droplet, respectively, $R$ is the droplet radius. Figure 1 shows the pressure difference against $1 / R$ with $\mathrm{A}=0.003, \kappa=0.01, \tau_{f}=0.8, \tau_{g}=1.0$ and $\Gamma=8.0$. The model predictions are in excellent agreement with the analytical solution. To assess the fluid-surface interaction model given by Eq. (27), we perform simulation in a $240 \times 240$ domain. The initial water "droplet" is defined as a square of $40 \times 40$ lattice cells on a solid wall. We run the simulation until the shape of droplet does not change at an equilibrium state. Figure 2 show the results using two different wetting boundary treatments for a droplet on a solid surface with the static contact angle $\theta=45^{\circ}, 90^{\circ}$ and $135^{\circ}$ respectively, where (a) uses the method described above, and (b) uses the method proposed by Briant et al. [23]. The results confirm that the current wetting boundary treatment is suitable to model the interaction between fluids and solids.

Taylor deformation is often used to assess the capability of multiphase model for a dynamic problem. A droplet is placed between two parallel plates which are moving in opposite directions to obtain linear shear in the Stokes regime (small Reynolds number), and droplet deformation is studied as a function of the shear rate (expressed as the Capillary number) at a constant Peclet number. The definitions of the Reynolds number, Capillary number, and Peclet number $[18,22]$ are given as

$$
\mathrm{Re}=\frac{\dot{\gamma} R^{2} \rho}{\eta}, \quad C a=\frac{\dot{\gamma} R \eta}{\sigma}, \quad P e=\frac{\dot{\gamma} R \xi}{M A}
$$

where $\dot{\gamma}=2 U / H$ is the shear rate, which is the velocity of moving wall divided by the channel height. $R$ is the radius of the droplet, and $\eta$ is the dynamic viscosity. For this case, we assume that the densities and viscosities are the same for both fluids. In order to reproduce correct physical behaviour of a droplet, 
simulations were run with $C h^{*}=\xi / \Delta x=1$ and 2, $R e=0.1$ and $P e=O(1)$. The droplet diameter is 64 lattice cells in a flow domain of $256 \times 128$ lattice cells. At the steady state, the droplet is assumed to be an elliptic shape, which is usually characterized by the deformation parameter $D f=(L-B) /(L+B), L$ and $B$ being the major and minor axis of the ellipse. The VOF simulations are also performed with the same physical parameters and gird size. In Figure 3 we have plotted $D f$ versus $C a$ for both LB and VOF simulations. We observe that the simulation results with $C h^{*}=2$ agree better with the VOF results [18] than simulation results with $C h^{*}=1$. Clearly, interface thickness influences the numerical results to some extent and have to be chosen with care. In addition, we find the droplet breaks up at the critical Capillary number $0.9<C a_{c r}<1.0$ for $C h^{*}=2$, which is in agreement with the finding of Zhou and Pozrikidis [24]. Figure 4 shows snapshots of the droplet breakup process under shear at $R e=1.0$ and $C a=1.4$. The dimensionless time is defined as $t^{*}=\dot{\gamma} t /(2 \mathrm{Ca})$. From the results, it can be expected the thicker interface is more suitable to describe droplet deformation and breakup. Hence, we choose $C h^{*}=2$ in the following simulations.

\subsection{Simulations of droplet formation in a $\mathbf{T}$ junction}

A T-junction microchannel with two inlets and single outlet is used in this study and the channel width $L$ is 100 microns. The continuous phase oil is introduced from the inlet of main channel, and the dispersed phase water is injected from the inlet of lateral channel. The Reynolds number, Capillary number and Peclet number are defined as Eq. (30) with $\dot{\gamma}=u_{c} / L, R=L$ and $\eta=\eta_{c}$, where $u_{c}$ and $\eta_{c}$ are the mean velocity and viscosity of continuous phase respectively. To describe the size of droplet formed in the channel, we introduce the rescaled droplet area $S=S_{d} / L^{2}$.

The simulations are performed in a computational domain with $300 \times 60$ cells and each cell corresponds to 5 microns. We have used an increased resolution with $600 \times 120$ cells and find that the numerical results are close to the above coarse mesh. Therefore, we will use the coarse mesh in the simulations below. The velocity and viscosity ratios of the continuous phase to the dispersed phase are fixed as $1 / 4$ and 1 respectively. We apply the boundary conditions as described in the section 2.3 . Since the continuous oil phase will form a thin layer at the channel wall, we assume that the continuous phase completely wets the wall surface while the dispersed phase is repelled except the cases studying the influence of wettability of fluids at the wall. For the flow regimes under consideration, the Reynolds number is not more than 1.0. We mainly focus on investigating the influences of Peclet number, Capillary number and contact angle on droplet formation. The influence of inlet velocity ratio and viscosity ratio can be found in [25].

First we investigate the effect of the Peclet number on droplet formation at different Capillary number. As seen in Eq.(30), the Peclet number is the ratio of the convection time scale and the interface diffusion time scale, which is directly related to the Cahn-Hilliard convection-diffusion equation. For a finite interface thickness, straining flows can thicken or thin the interface, leading to numerical "Marangoni effect" [26], which must be resisted by diffusion. On the other hand, too large diffusion will excessively damp the flow. Therefore, the Peclet number must be appropriately chosen [27]. Simulations are performed with $u_{c}=0.001$ and $\eta_{c}=0.1$. The Capillary number varies from 0.0025 to 0.025 , typically found in microfluidic droplet generation. The Peclet number is varied between $0.5 \leq P e \leq 10$, which was stressed by van der Sman et al. [18] that the interface of droplet evolves in a reasonably physical manner by examining a droplet deformation under shear flow. Figure 5 shows the flow patterns at different $C a$ for $P e=0.5,5$ and 10 respectively. We find that the Peclet number can slightly affect the timing of droplet formation, and thus has small effect on droplet size. Figure 6 plots the rescaled droplet area as a logarithm function of the Capillary number at different $P e$. For a fixed $P e$, the droplet size always decreases as $C a$ increases. However, the trends of variation appear to be different for the Peclet number considered: $P e=0.5$ and 1 give a nearly linear variation; $P e=5$ shows a slight variation in slope but not obvious; $P e=10$ gives a clear variation in slope and two distinct droplet generation regimes are clearly identified. When the magnitude of Peclet number is further increased, we find the interface thickness shows highly uneven around the droplet. Comparing the above findings with the previous experimental and numerical results, we can easily find that $P e=10$ can produce a reasonable droplet behaviour for the droplet formation in a T-shaped microchannel. The squeezing-to-dripping transition is well captured at $P e=10$ by the current model with a critical Capillary number $C a_{c} \approx 0.012$, which was also reported in experiment [4] with $C a_{c} \approx 0.01$ and simulation [25] with $C a_{c} \approx 0.015$. 
To investigate the influence of the Capillary number on droplet formation, three parameters included in $C a$, i.e. viscosity, inlet velocity and interfacial tension, are studied individually by fixing the other two. A series of simulations are performed with (a) $u_{c}=0.001, \sigma=0.008$ and $\eta_{c}$ varying from 0.02 to 0.2 , (b) $\sigma=0.008, \eta_{c}=0.1$ and $\eta_{c}$ varying from 0.0002 to 0.002 , and (c) $u_{c}=0.001, \eta_{c}=0.1$ and $\sigma$ varying from 0.004 to 0.04 . Figure 7 shows the flow patterns for various parameters at $\dot{\gamma} t=10$. When $\mathrm{Ca}$ increases, the distance between two neighboring droplets decreases and the size of generated droplets also decreases. Figure 7 shows that the shape and size of the droplets are almost the same for each $\mathrm{Ca}$, although the other dimensionless number (e.g. $\mathrm{Re}$ ) is different. So $\mathrm{Ca}$ dominates the droplet formation to a large extent under the current conditions. However, Figure 8 shows that adjusting viscosity to schieve the same $\mathrm{Ca}$ leads to result deviation from those adjusting velocity and interfacial tension in the squeezing regime (low $\mathrm{Ca}$ ). $\mathrm{Wu}$ et al. [28] also reported the similar findings in the simulation of a cross-junction microchannel. They argued that, this may be because the Weber number (the product of the Capillary number and the Reynolds number) also influences the flow simultaneously although its effect is much smaller than the Capillary number under the current conditions. To understand the mechanicsm of the deviation, we run simulations in the squeezing regime and keep the same $C a, R e$ and We (Weber number), $\left(u_{c}=0.0004, \eta_{c}=0.08\right.$ and $\sigma=0.008$, and $u_{c}=0.0008, \eta_{c}=0.16$ and $\sigma=0.032$ ). Surprisingly, the formed droplet sizes are different with $S=1.19,1.15$ respectively. The good agreement between the velocity variation and the interfacial tension variation indicates that $W e$ nor $R e$ has no effect on droplet size under the current conditions (the squeezing and creeping flow regime). On the contrary, the viscosity palys a special role during the droplet formation in the squeezing regime. It may affect the built-up of upstream pressure due to the obstruction of the main channel by the emerging droplet, which is important for the droplet formation process in the squeezing regime.

Due to high surface to volume ratio, fluid/surface interaction will significantly influence the droplet dynamics in microfluidic devices. Figure 9 shows the flow patterns at various $\mathrm{Ca}$ for three different wetting properties $\left(\theta=120^{\circ}, 150^{\circ}\right.$ and $\left.180^{\circ}\right)$. Obviously, the wetting conditions influence the position of droplet detachment, droplet shape and size, especially under less hydrophobic wetting conditions $\left(\theta=120^{\circ}\right)$. The position of droplet detachment moves towards downstream as $C a$ increases for small contact angle. At the same time, we can observe the phase interfaces are prone to normal to the solid walls at small contact angles, which is in accordance with the theory of interface dynamics. In addition, we find that the advancing and receding contact angles are different and both deviate from the specified equilibrium contact angle $\theta$ for the dynamic droplet in motion. This phenomenon has been observed in many experiments, and is well known as the contact angle hysteries [29]. Figure 10 gives the rescaled droplet area $S$ as a function of $C a$ for different contact angles. It can be found that more hydrophobic wetting condition is generally expected to produce smaller droplet. Wettability has large effect on droplet size for small $\mathrm{Ca}$, but its effect gradually deceases when $\mathrm{Ca}$ increases. The reason may be that the size of generated droplet at a small $\mathrm{Ca}$ is usually larger than that at a high $\mathrm{Ca}$, which leads to larger contact area with the solid wall. Therefore, the enhanced interaction with solid wall affects the droplet behaviour more significantly. It is also observed that the droplet size becomes quickly independent of $\mathrm{Ca}$ when $\mathrm{Ca}$ decreases for $\theta=150^{\circ}$, which was also experimentally reported by Garstecki et al. [4]

\section{CONCLUSIONS}

Free energy lattice Boltzmann model has been used to simulate immiscible fluids in microfluidic devices. An improved stress-free boundary condition is proposed to treat multiphase outlet boundaries, which can conserve the total mass of the system and improves the stability of computation. The influences of Peclet number, Capillary number and contact angles on droplet formation in a $\mathrm{T}$-junction channel have been studied. The flow patterns and generated droplet size are dominated by the Capillary number when $\operatorname{Re} \leq 1$ for a fixed viscosity. The wetting properties can affect the droplet shape and size, the position of detachment, and its effect becomes more significant when the Capillary number decreases. It is expected that more hydrophobic wetting condition can produce smaller droplet. 


\section{REFERENCES}

[1] Joanicot, M., \& Ajdari, A. (2005). Droplet control for microfluidics. Science, 309, 887-888.

[2] Kelly, B. T., Baret, J. C., Talyab, V., \& Griffiths, A. D. (2007). Miniaturizing chemistry and biology in microdroplets. Chem. Commun., 18, 1773-1788.

[3] Thorsen, T., Roberts, R. W., Arnold, F. H., \& Quake, S. R. (2001). Dynamic pattern formation in a vesicle-generating microfluidic device. Phys. Rev. Lett., 86, 4163-4166.

[4] Garstecki, P., Fuerstman, M. J., Stone, H. A., \& Whitesides, G. M. (2006). Formation of droplets and bubbles in a microfluidic T-junction-scaling and mechanism of break-up. Lab Chip, 6, 437-446.

[5] Shyy, W., Udaykumar, H. S., Rao, M. M., \& Smith, R. W. (1996). Computational fluid dynamics with moving boundaries. London: Taylor \& Francis.

[6] Succi, S. (2001). The lattice Boltzmann equation for fluid mechanics and beyond. Oxford: Oxford University Press, Clarendon Press.

[7] He, X., \& Luo, L-S. (1997). A priori derivation of the lattice Boltzmann equation. Phys. Rev. E, 55, R6333-R6336.

[8] Gunstensen, A., Rothman, D. H., Zaleski, S., \& Zanetti, G. (1991). A lattice Boltzmann model of immiscible fluids. Phys. Rev. A, 43, 4320-4327.

[9] Shan, X., \& Chen, H. D. (1993). Lattice Boltzmann model for simulating flows with multiple phases and components. Phys. Rev. E, 47, 1815-1819.

[10] Swift, M. R., Osborn, W. R., \& Yeomans, J. M. (1995). Lattice Boltzmann simulation of nonideal fluids. Phys. Rev. Lett., 75, 830-833.

[11] Swift, M. R., Orlandini E., Osborn, W. R., \& Yeomans, J. M. (1996). Lattice Boltzmann simulations of liquid-gas and binary fluid systems. Phys. Rev. E, 54, 5041-5052.

[12] He, X., Doolen, G. D. (2002). Thermodynamic foundations of kinetic theory and lattice Boltzmann models for multiphase flows. J. Stat. Phys., 107, 309-328.

[13] Chikatamarla, S. S., Ansumali, S., \& Karlin, I. V. (2006). Grad's approximation for missing data in lattice Boltzmann simulations. Europhys. Lett., 74(2), 215-221.

[14] Tong, C. Q., He, Y. L., Tang, G. H., Wang, Y., \& Liu, Y. W. (2007). Mass modified outlet boundary for a fully developed flow in the lattice Boltzmann equation. Int. J. Mod. C, 18(7), 1209-1221.

[15] Aiduny, C., Lu, Y., \& Ding, E. J. (1998). Direct analysis of particulate suspensions with inertia using the discrete Boltzmann equation. J. Fluid Mech., 373, 287-311.

[16] Qian, Y. H., d'Humieres, D., \& Lallemand, P. (1992). Lattice BGK models for Navier-Stokes equation. Europhys. Lett., 17(6), 479-484.

[17] Polley, C. M., \& Furtado, K. (2008). Eliminating spurious velocities in the free-energy lattice Boltzmann method. Phys. Rev. E, 77, 046702.

[18] van der Sman, R. G. M., \& van der Graaf, S. (2008). Emulsion droplet deformation and breakup with lattice Boltzmann model. Comput. Phys. Comm., 178, 492-504.

[19] Jacqmin, D. (1999). Calculation of two-phase Navier-Stokes flows using phase-field modeling. $J$. Comput. Phys., 155, 96-127.

[20] Ladd, A. J. C. (1994). Numerical simulations of particulate suspensions via a discretized Boltzmann equation (Parts I \& II). J. Fluid Mech., 271, 285-339.

[21] Iwahara, D., Shinto, H., Miyahara, M., \& Higashitani, K. (2003). Liquid droplets on homogeneous and chemically heterogeneous surfaces: a two-dimensionla lattice Boltzmann study. Langmuir, 19, 90868093.

[22] van der Graaf, S., Nisisako, T., Schroen, C., van der Sman, R. G. M., \& Boom R. M. (2006). Lattice Boltzmann simulations of droplet formation in a T-shaped microchannel. Langmuir, 22, 4144-4152.

[23] Briant, A. J., \& Yeomans, J. M. (2004). Lattice Boltzmann simulations of contact line motion. II. Binary fluids. Phys. Rev. E, 69, 031603.

[24] Zhou, H., \& Pozrikidis, C. (1993). The flow of suspensions in channel: single file of drops. Phys. Fluids, 5(2), 311-324.

[25] de Menech, M., Garstecki, P., Jousse, F., \& Stone, H. A. (2008). Transition from squeezing to dripping in a microfluidic T-shaped junction. J. Fluid Mech., 595, 141-161.

[26] Yu, W., \& Zhou, C. (2007). Coalescence of droplets in viscoelastic matrix with diffuse interface under simple shear flow. J. Polym. Sci., Part B: Polym. Phys., 45(14), 1856-1869.

[27] de Menech, M. (2006). Modeling of droplet breakup in a microfluidic T-shaped junction with a phasefield model. Phys. Rev. E, 73, 031505.

[28] Wu, L., Tsutahara, M., Kim, L. S., \& Ha, M. Y. (2007). Numerical simulations of droplet formation in a cross-junction microchannel by the lattice Boltzmann method. Int. J. Numer. Meth. Fluids, 57(6), 793810. 


\section{FIGURES}

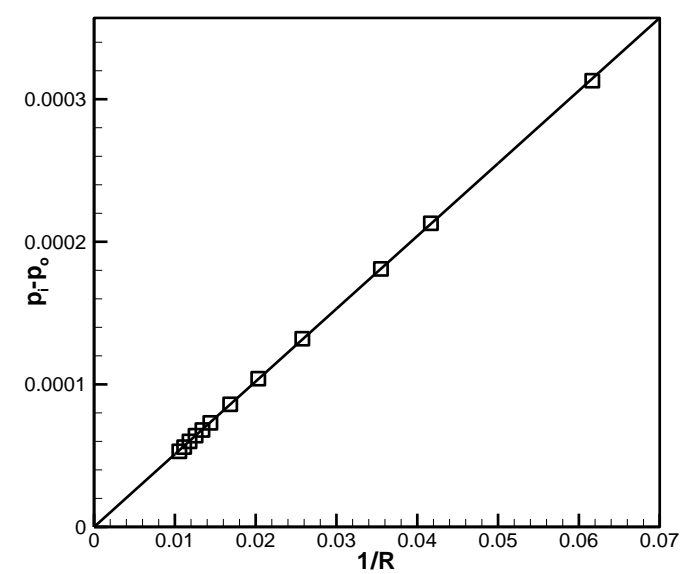

Figure 1: Numerical verification of the Laplace's law. The symbols $\square$ represent the simulation results of our LB model and the solid line is the analytical solution of Eq. (29).

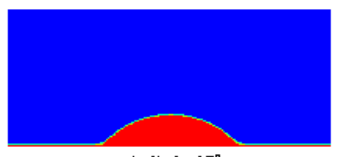

(a1) $\theta=45^{a}$

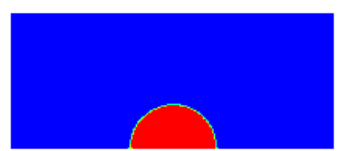

(a2) $\theta=90^{\circ}$

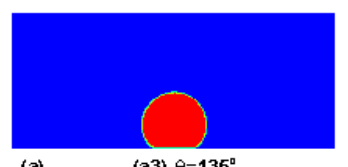

(a3) $\theta=135^{\circ}$

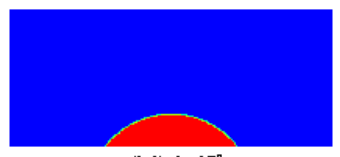

(b1) $\theta=45^{\circ}$

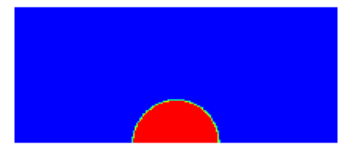

(b2) $\theta=\mathbf{9 0}$

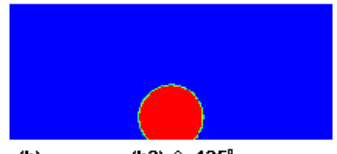

(b3) $\theta=135^{\circ}$

Figure 2: The simulation of static contact angles using (a) the present method and (b) Briant's method [23].

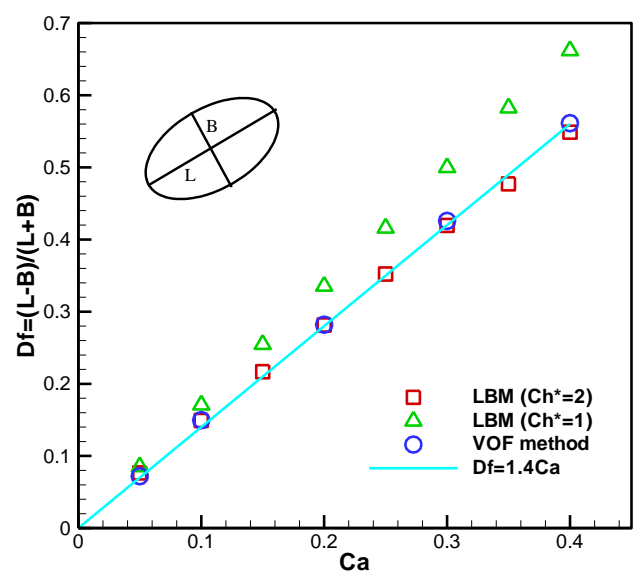

Figure 3: Comparison of droplet deformation between our lattice Boltzmann model (LBM) and the VOF method where $R e$ is 0.1 . 


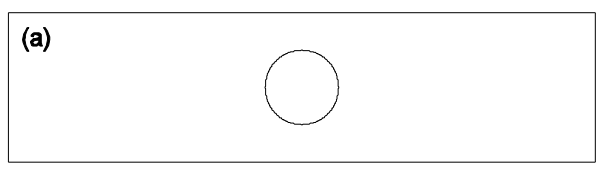

(c)

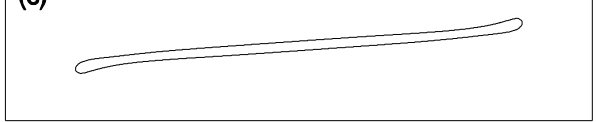

(e)

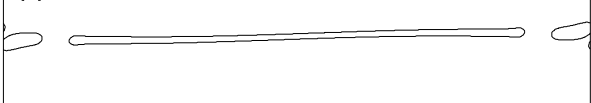

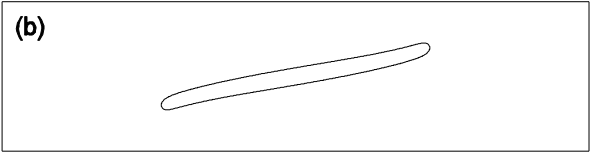

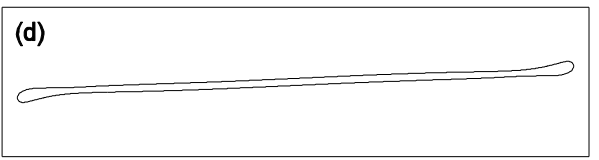

(f)

Figure 4: Snapshots of droplet breakup under shear, with $R e=1.0, C a=1.4$ and $C h^{*}=2$, are recorded at: (a) $t^{*}=0$, (b) $t^{*}=2$, (c) $t^{*}=4$, (d) $t^{*}=6$, (e) $t^{*}=8$, and (f) $t^{*}=10$.

(a)
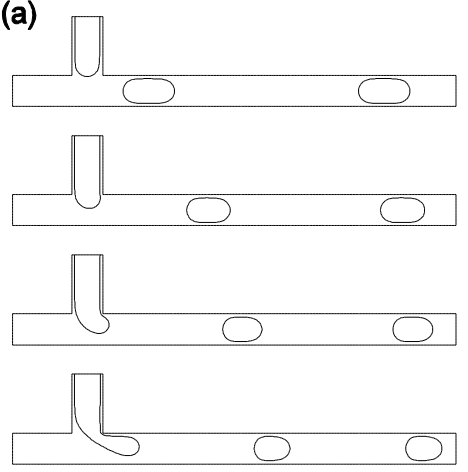

(b)
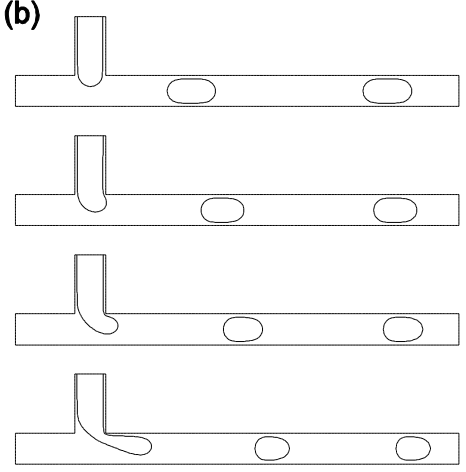

(c)

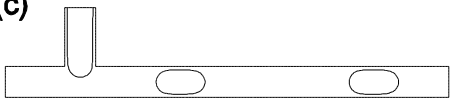

$\mathrm{Ca}=0.002$

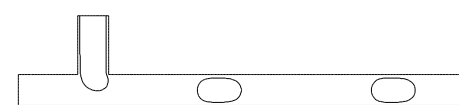

$\mathrm{Ca}=0.008$

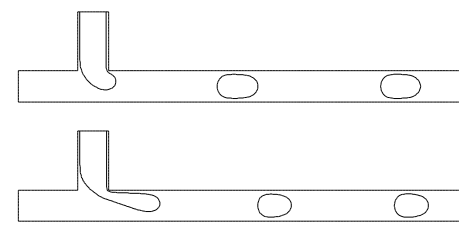

$\mathrm{Ca}=0.015$

$\mathrm{Ca}=0.025$

Figure 5: The flow patterns at $\dot{\gamma} t=10$ for various $C a$ with the Peclet number: (a) $P e=0.5 ; \quad$ (b) $P e=5$; and (c) $P e=10$.

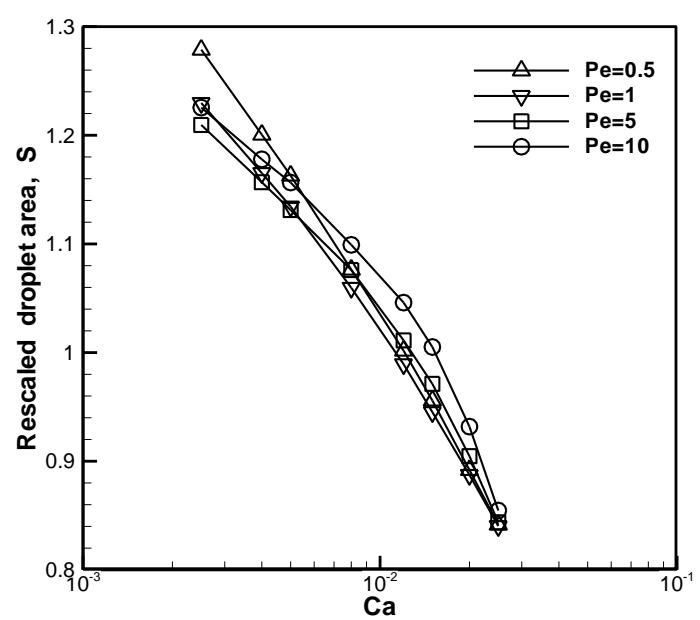

Figure 6: The rescaled droplet area $S$ as a function of $C a$ for four different $P e$. 
(a)
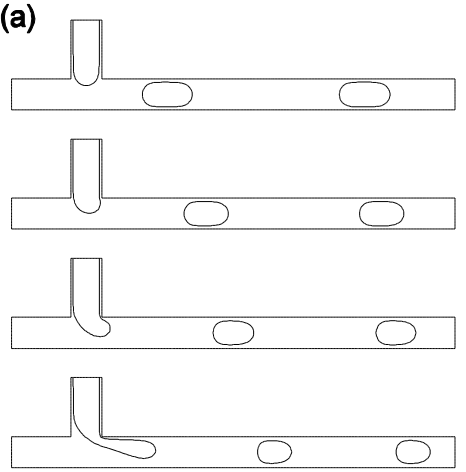

(b)
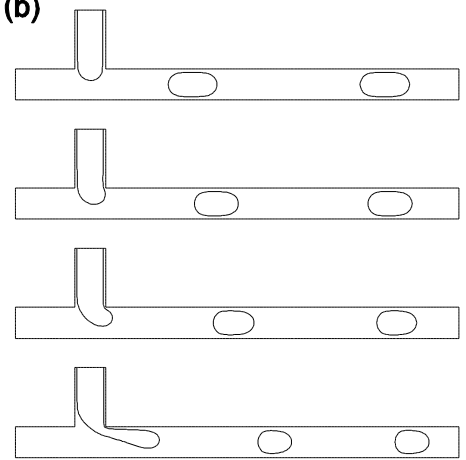

(c)

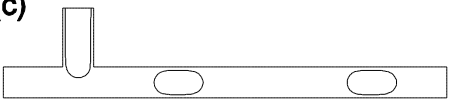

$\mathrm{Ca}=0.002$

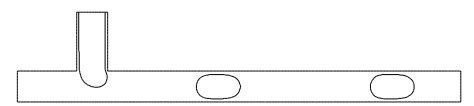

$\mathrm{Ca}=0.008$

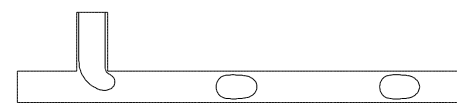

$\mathrm{Ca}=0.015$

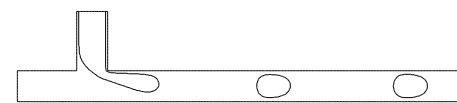

$\mathrm{Ca}=0.025$

Figure 7: The flow patterns forincreasing $C a$ at $\dot{\gamma} t=10$ by adjusting: (a) $\eta_{c}$; (b) $u_{c}$; and (c) $\sigma$.

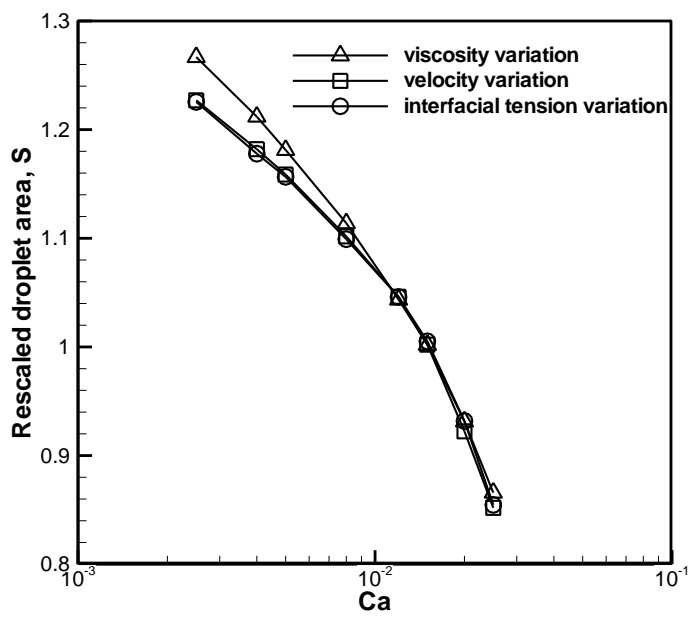

Figure 8: The rescaled droplet area at different $C a$ by adjusting parameters $\eta_{c}, u_{c}$ and $\sigma$.

(a)
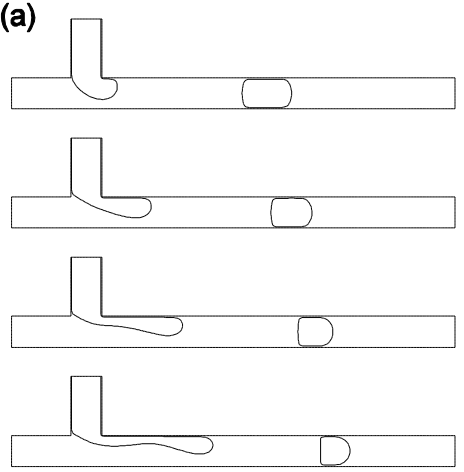

(b)
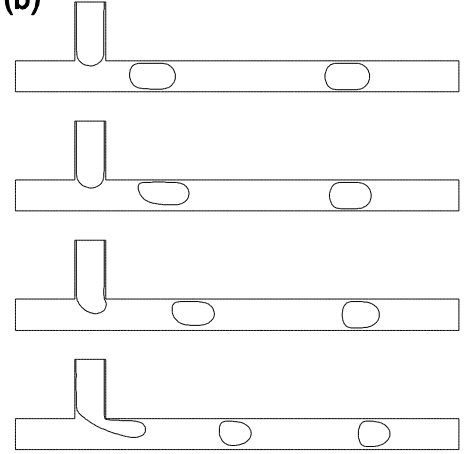

(c)

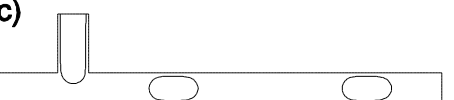

$\mathrm{Ca}=0.002$

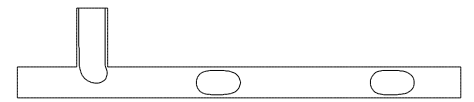

$\mathrm{Ca}=0.008$

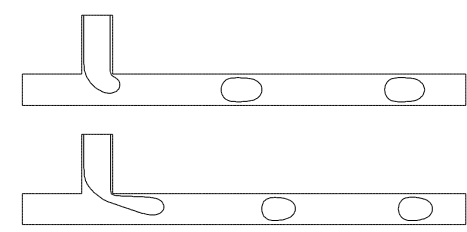

$\mathrm{Ca}=0.015$

$\mathrm{Ca}=0.025$

Figure 9: The flow patterns for various $C a(\dot{\gamma} t=10)$ with the contact angles: (a) $\theta=120^{\circ}$; (b) $\theta=150^{\circ}$ and (c) $\theta=180^{\circ}$. 


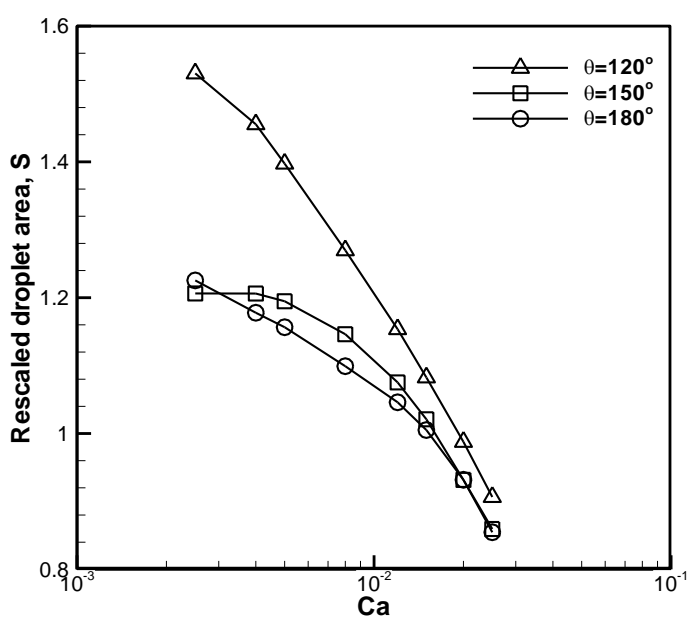

Figure 10: Rescaled droplet area $S$ as a function of $C a$ for three different contact angles. 